$10-15-2018$

\title{
StormSense: A Blueprint for Coastal Flood Forecast Information \& Automated Alert Messaging Systems
}

Jon Derek Loftis

VIMS

Sridhar Katragadda

City of Virginia Beach

Sokwoo Rhee

National Institute of Standards and Technology

Cuong Nguyen

National Institute of Standards and Technology

Follow this and additional works at: https://scholarworks.wm.edu/vimsarticles

Part of the Oceanography Commons

\section{Recommended Citation}

Loftis, Jon Derek; Katragadda, Sridhar; Rhee, Sokwoo; and Nguyen, Cuong, "StormSense: A Blueprint for Coastal Flood Forecast Information \& Automated Alert Messaging Systems" (2018). VIMS Articles. 1239. https://scholarworks.wm.edu/vimsarticles/1239 


\title{
StormSense: A Blueprint for Coastal Flood Forecast Information \& Automated Alert Messaging Systems
}

\author{
Jon Derek Loftis \\ Virginia Institute of Marine Science, \\ College of William \& Mary \\ Gloucester Point, VA, U.S.A. \\ e-mail: jdloftis@vims.edu
}

\author{
Sridhar Katragadda \\ City of Virginia Beach, \\ Dept. of Comm. and Info. Technology \\ Virginia Beach, VA, U.S.A. \\ e-mail: SKatraga@,vbgov.com
}

\author{
Sokwoo Rhee and Cuong Nguyen \\ Smart Grid \& Cyber-Physical Systems Program Office, \\ National Institute of Standards and Technology \\ Gaithersburg, MD, U.S.A. \\ e-mails: sokwoo.rhee@,nist.gov \\ \& cuong.nguyen@nist.gov
}

\begin{abstract}
Increased availability of low-cost water level sensors communicating through the Internet of Things (IoT) has expanded the horizons of publicly-ingestible data streams available to modern smart cities. StormSense is an IoT-enabled inundation forecasting research initiative and an active participant in the Global City Teams Challenge seeking to enhance flood preparedness in the smart cities of Hampton Roads, VA for flooding resulting from storm surge, rain, and tides. In this study, we present the a blueprint and series of applicable protocols through the use of the new StormSense water level sensors to help establish a regional resilience monitoring network. In furtherance of this effort, the Virginia Commonwealth Center for Recurrent Flooding Resiliency's Tidewatch tidal forecast system is being used as a starting point to integrate the extant (NOAA) and new (USGS and StormSense) water level sensors throughout the region, and demonstrate replicability of the solution across the cities of Newport News, Norfolk, and Virginia Beach within Hampton Roads, VA. StormSense's network employs a mix of ultrasonic sonar and radar remote sensing technologies to record water levels and develop autonomous alert messaging systems through the use of three separate cloud environments. One to manage the water level monitoring sensors and alert messaging, one to run the model and interface with the post-processed results, and one to geospatially present the flood results.
\end{abstract}

Keywords-Hydrodynamic Modeling, Internet of Things, Smart City, Global City Teams Challenge, Replicability, Citizen Science, Sea Level Rise

\section{INTRODUCTION}

Modern smart cities are functionally equivalent to a complex system. These systems are often subjected to many non-linear influences on how to efficiently allocate their limited resources [1]. The protocols used to determine how smart cities respond to emergency flooding conditions in the future could be adapted using models. These models should be informed and validated by a dense water level sensor network to most efficiently advise how best to prepare for the imminent flood-related disasters of the future [2,3]. As many data-driven projects are afforded greater versatility on cloud based platforms, StormSense approaches flood monitoring and modeling via the cloud. This is done through the use of IoTsensor monitoring, online mapping, and through predictive tidal and hydrodynamic modeling with automated alerts [4].

StormSense is a flood prediction project initiated by the proactive local governments in tidewater Virginia. It detects, models, and communicates flood risk with help from scientists at the Virginia Institute of Marine Science (VIMS) and partner city engineers via IoT water level sensors, hydrodynamic models, artificial intelligence, and voice-assisted technologies. StormSense operates and disseminates flood forecasts via a

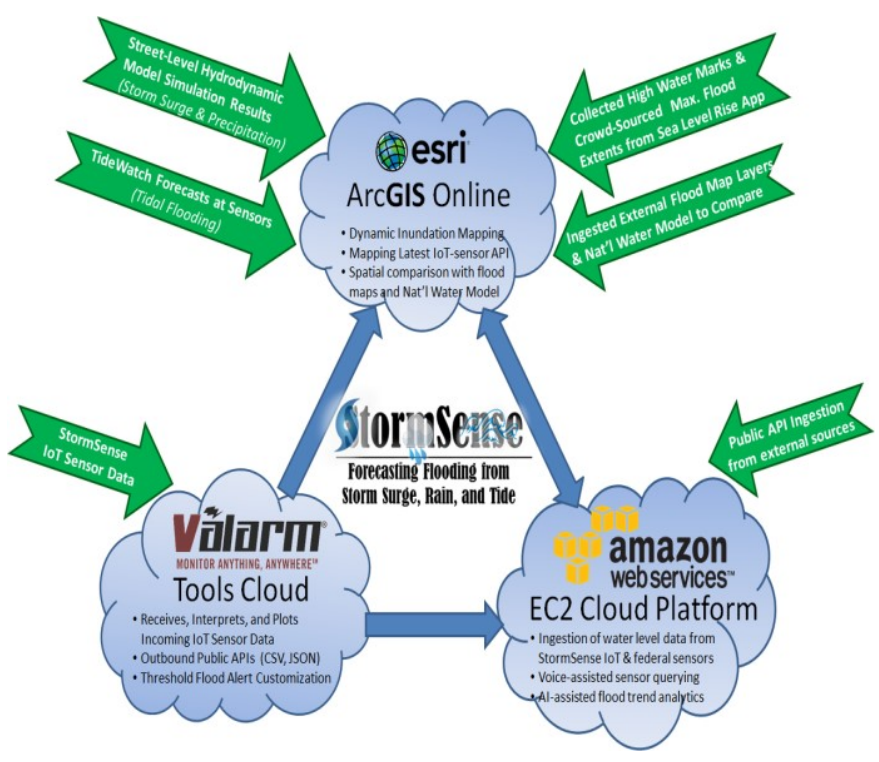

Figure 1. StormSense's triumvirate of cloud platforms employed for automated flood alert messaging. Inputs noted in green arrows, with blue arrows depicting exchange between cloud platforms. Click figure for larger view. 
triumvirate of cloud platforms operated by Valarm, ESRI, and Amazon Web Services (AWS) (Figure 1):

1) Valarm's IoT water level sensors densify the existing model data matrix and help us better understand the varying wind conditions that cause recurrent ephemeral flooding.

2) AWS' cloud platform aids with smart voice-assisted technologies using Reverb/Amazon Alexa, to place flood observations and predictions in citizens' hands visibly and audibly via their smart devices and StormSense's AWS.

3) ESRI's ArcGIS Online mapping environment visually disseminates flood model forecasts. Citizens find that flood layers overlapping their house, driveway, or route to work, is difficult to misinterpret.

The computationally-intensive nature of the hydrodynamic modeling approach is such that StormSense currently operates with the limitation that storm surge and heavy rainfall forecasts require the model to be manually submitted for simulation via high performance computing platfo rms or on AWS' EC2 cluster [4]. However, tidal flooding forecasts are automated through a service VIMS operates called Tidewatch. Hydrodynamic modeling of storm surge requires the implementation of a large-scale regional model to accurately capture the large scale wind influence of hurricanes and nor'easters as their storm surges transition from the open ocean to Atlantic Shelf, into Chesapeake Bay and then into its contributing estuaries. Heavy rainfall events are complex to model, partially because precipitation observation data for the region are currently logged in an aging system architecture by semi-private regional entities, and also due to lack available

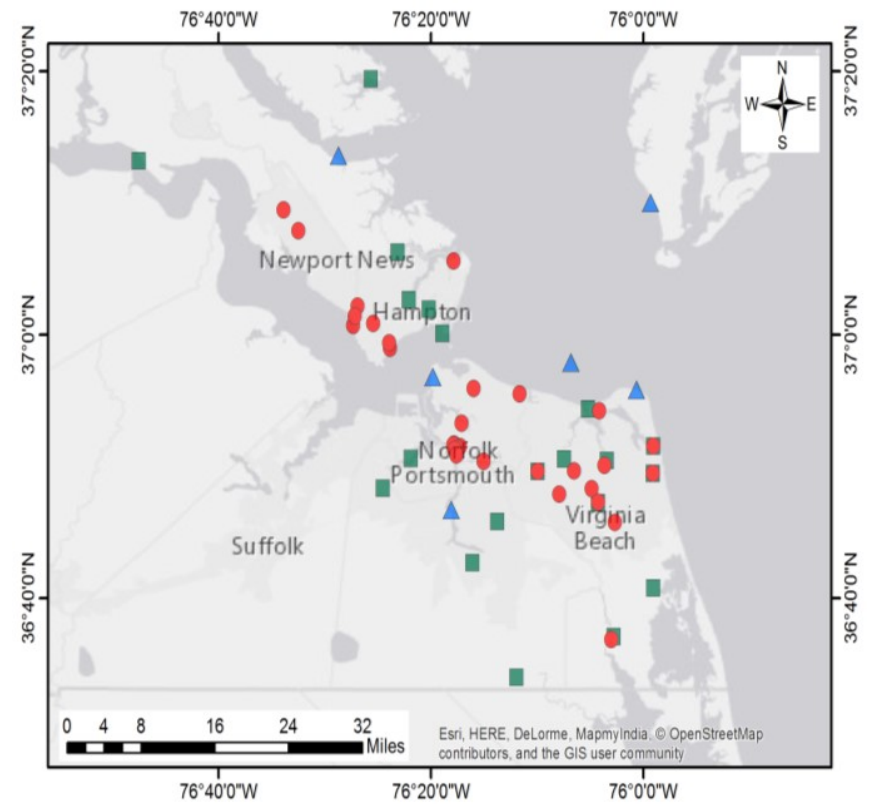

Figure 2. Map of 57 publicly-streaming water level monitoring stations throughout Hampton Roads, VA. The StormSense sensor network has contributed 28 sensors to the 29 existing sensors maintained by federal entities. Of these, NOAA has 6 (marked in blue) and USGS maintains 19 (noted in green). Additionally, VIMS has 1, and WeatherFlow has 3 (also marked in red). Click figure or http://arcg.is/14aCe1 for interactive map. higher-order temporal and spatial resolution data to most accurately forecast heavy precipitation events [5].

Despite these limitations, the most frequent form of flooding experienced by the localities in Hampton Roads is tidal nuisance flooding [6]. Alert messaging for tidal flooding events can be addressed through a completely automated approach which can make use of sensors and harmonic tidal signature extraction techniques. These methods can be harnessed to estimate when tidal flooding is likely to occur at or near a sensor, and automate alerts associated with designated flood thresholds as an automated and advanced early warning system desired by coastal smart cities to protect citizens, infrastructural assets, and qualify for decreases in flood insurance premiums proportional to the alert system's advance warning time and sophistication. As Tidewatch currently provides tide forecasts up to 36 hours in advance of tidal inundation events, this approach is desirable for smart communities participating in FEMA's National Flood Insurance Program.

Thus, the StormSense Project brings together municipal governments in Hampton Roads, Virginia, including: Newport News, the RSCT grant recipient, Norfolk, Virginia Beach, Hampton, Chesapeake, Portsmouth, Williamsburg, and York County along with VIMS, to develop a regional resilience monitoring network [6]. This network of 28 newly-installed publicly-broadcasting water level sensors ingests and interfaces with open Application Programing Interface (API) data from federal monitoring and water prediction agencies (such as USGS and NOAA) to bring the total number of water level sensors to 57 (Figure 2). With most of these sensors being recently installed in 2016-2017, StormSense is poised to develop the network as Phase 1 [7], and develop a street-level flood forecasting and monitoring solution across the entire region for Phase 2 [4], which begins with integration of observed water-levels into VIMS' Tidewatch tidal forecasting system, which now operates under the Virginia Commonwealth Center for Recurrent Flooding Resiliency (CCRFR) at: https://www.floodingresiliency.org/ [3].

Many existing smart cities solutions are designed to have a measurable impact on specific key performance indicators relevant to their communities. Since many of today's smart city/community development efforts are often isolated and highly customized projects, the National Institute of Standards and Technology (NIST) launched the Global City Teams Challenge (GCTC) to encourage collaboration and the development of standards for smart cities. The GCTC's longterm goal is to demonstrate a scalable and replicable model for incubating and deploying interoperable, adaptable, and configurable Internet of Things (IoT)/Cyber-Physical Systems technologies in smart cities/communities [1]. This program aims to help communities benefit from working with others to improve efficiency and lower costs. NIST also created the Replicable Smart City Technology (RSCT) cooperative agreement program to provide funding to enable awardee City/Community Partners to play a lead role in the team-based 
GCTC effort to pursue measurement science for replicable solutions [8]. The RSCT program was designed to support standards-based platform approaches to smart cities technologies that can provide measurable performance metrics. Together these two programs work to advance state-of-the-art smart city standards. The city of Newport News was awarded an RSCT grant in September 2016 on behalf of the StormSense Project's application to NIST. Thus, the implementation of open-source models, accessible cloud platforms, and low-cost IoT solutions ideally embody the GCTC mantra by make StormSense's solution tenably replicable, scalable, and measurable [7]. The combined nature of this approach ideally will not only make a difference in our region, but potentially in other flood-prone regions of the world through the use of the blueprint presented in the next section and the data ingestion protocols noted throughout this paper.

\section{STUDY AREA AND MODEL BLUEPRINT}

Hampton Roads, VA, is the second-largest population center at risk from sea level rise in the United States. The region has more than 400,000 properties that are exposed to flood or storm surge inundation [9]. The region has a population of over 1.7 million people, living and traveling on roads exposed to both severe and increasing frequent chronic "nuisance" flooding [10,11]. A major issue Hampton Roads faces is that the region experiences nuisance flooding fatigue with such frequency that it is easy to forget that flooding events cost our cities, their first responders, and their residents time and money [12]. In one neighborhood in the City of Newport News particularly prone to nuisance flooding, typically many emergency responders were required to assist in evacuating the complex $[13,14]$. However, by remotely alerting residents that the water was rising quickly on the local stream, the past two flooding events have not required any emergency responders to assist in evacuating and were subsequently able to dedicate their emergency services elsewhere $[14,15]$. The goal of establishing a flood monitoring network can be expensive, but in the long term, the anticipated benefits of improved quality of life for a region's citizens are monumental. The goal is to replicate this level of success throughout the cities of Hampton Roads by providing a greater density of water level sensors. As an added benefit, more publicly-available water level sensors empower property owners to take responsibility for their assumed risk of living adjacent to floodplains. This has resulted in a marked spike in the number of residents who have opted for flood insurance, with 2,231 claims totaling \$25M in damage attributed to 2016 Hurricane Matthew [5,16]. Many of these properties are insured through the Federal Emergency Management Agency's (FEMA) National Flood Insurance Program, but many properties outside of the designated floodplain do not have preferred risk policies $[12,16]$. Thus, StormSense has developed a blueprint which has been shared with the GCTC community via the Public Safety Supercluster at the 2017 GCTC Expo in Washington, D.C. (Figure 3)[17].

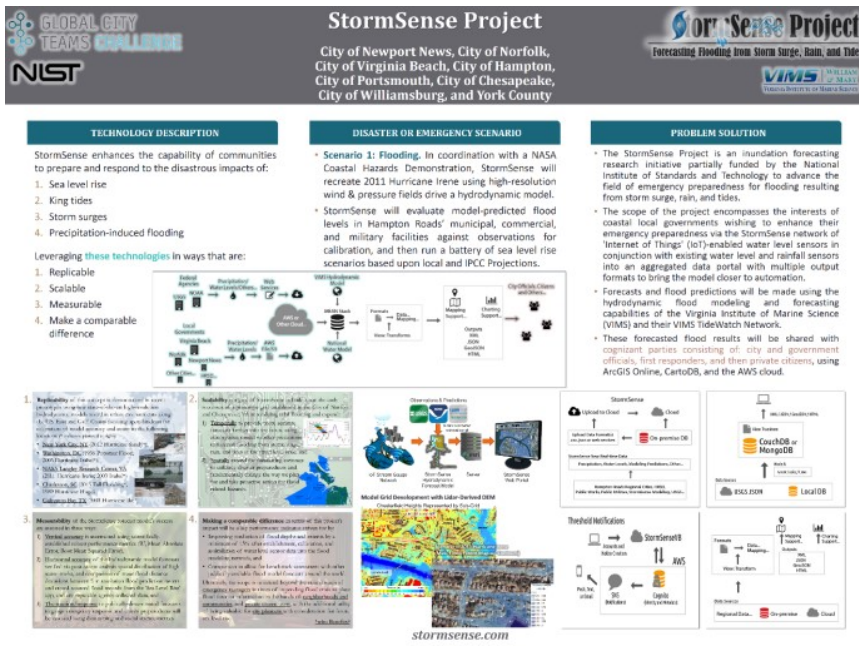

Figure 3. GCTC Blueprint Solution for StormSense flood monitoring, model predictions, and automated alert system protocols. Click figure for larger view.

Existing flood communication and messaging systems have not yet responded to the changing risk patterns brought by sea level rise and have not been able to meet the diverse needs of a growing populous in an expanding floodplain. Thus, a better understanding of flood risk perception, information seeking behavior and decision-making can inform the development of new communications tools and flood risk messaging [18]. This is the percieved intersect between new IoT-technologies and emerging flood model validation methods. For each storm event, in Hampton Roads, water levels driven via 36-hour Tidewatch forecasts provided by VIMS at NOAA's Sewells Point gague are typically used to drive surge and tides in urban-scale models. Now, forecasts from any of Tidewatch's ingested data from StormSense sensors can be used as the model's boundary conditions alongside wind and pressure inputs used to drive the model atmospherically, similar to Loftis, Wang, and Forrest [19]. VIMS employs a street-level hydrodynamic model, which incorporates a non-linear solver and variable sub-grid resolutions [2], capable of being embedded with lidar-derived topography to scale resolution for inundation where it is needed down to 5-m or even 1-m resolution in known areas where flooding frequency is high $[19,20]$. The model has been used to simulate every major storm event in Hampton Roads that has occurred in the past 25 years, and has been used in many other places along the U.S. East and Gulf Coasts as well [5,21-24]. For more information on the hydrodynamic models, please refer to these cited studies.

\section{WATER LEVEL SENSORS}

StormSense has recently deployed 28 bridge-mounted IoTultrasonic and microwave radar water level sensors in Newport News, Virginia Beach, and Norfolk, as outlined on the StormSense project's website at: http://www.stormsense.com. These sensors will complement the previously installed array of 6 gauges operated by NOAA, 19 relatively new gauges 
installed in 2015-2016 via Hurricane Sandy relief funds operated by the USGS, and 1 gauge operated by VIMS in Hampton Roads (Figure 2). While the extant remote sensors in the region are largely $\mathrm{K}_{\mathrm{a}}$-band radar sensors transmitting data through satellite signals, the new StormSense IoT-sensors enlist the use of ultrasonic sensors and transmit data via cellular transmission protocols or Long Range (LoRa) Wireless Area Networks (WAN), with the focus of creating a replicable costeffective network of sensors. Some realized utilities for a dense network of water level sensors are noted as follows:

1) Archiving water level observations for flood reporting

2) Validation/inputs for hydrodynamic flood models

3) Automated targeted advance flood alert messaging

4) Reliable interpolation of continuous water surface elevations throughout geo-event processing capabilities

\section{A. Water Level Sensor Types and Applications}

A collaboration between VIMS and the partner cities of: Newport News, Hampton, Norfolk, Virginia Beach, Portsmouth, Chesapeake, Williamsburg, and York County, in Hampton Roads, VA, will provide a prototype for strengthening emergency response times by providing spatial flood extent predictions in interactive map form at $5-\mathrm{m}$ resolution. The plan for integrating the inundation model into a more permanent warning system involves planned connection with the new sensors to the cities' current Everbridge notification systems for alert messaging. This occurs when the sensor observes flooding at user-specified elevations, and integration with model predictions for timely forecasted tidal inundation alerts through Tidewatch once the sensors are tidally-calibrated. In Newport News, the city employed a mix of $2 \mathrm{~K}_{\mathrm{a}}$-band radar sensors and 6 ultrasonic sonar sensors from Valarm, a California-based sensor vendor with a cloud-based virtual alarm messaging platform. The Valarm Tools Cloud platform uses the newly-installed sensors to provide subscriber-based alerts (Figure 4) based upon water level observations. The system will also eventually ingest tidal forecast predictions once incorporated into Tidewatch to enable cities to provide a unique flood-preparedness service to their citizens. An added benefit to this automated flood alert messaging method is that it can bolster the flood warning portion of their FEMA NFIP application to participate in the Community Rating System (CRS). This is important, as each higher participation level the city achieves in the hierarchical CRS program is commensurate with an additional 5\% decrease in flood insurance premiums for the citizen homeowners in participating communities.

StormSense demonstrates the benefits of replicating shared smart city solutions across multiple cities and communities that are facing similar flood challenges and it aligns with the goals of GCTC and RSCT programs [8]. For a different innovative example, Norfolk's LoRaWAN ultrasonic sensor network was established in city's historic Hague region in August 2017. The sensor network is currently comprised of one tide monitoring sensor mounted over The Hague walking bridge near where the USGS mounts their temporary rapid deployment gauge, and five inundation sensors, strategically positioned over frequently flooded streets [4,6]. The LoRaWAN sensors were purchased through a Norfolk-based vendor, GreenStream, Inc., and use long range WiFi instead of cellular data transmissions. They are currently publicly reporting water level observations in Tidewatch, and public API URLs are available at: http://www.vims.edu/people/ loftis_jd/HRVASensorAssets/index.php.

The recent installation of water level sensors provided by the USGS were used as an opportunity to demonstrate some of the benefits of added water level sensors using these ultrasonic sensors will be evaluated as reputable and replicable monitoring methods after a longer-term study. In pursuit of this, Figure 5 shows three examples of temporary StormSense ultrasonic sensors deployed on the same bridges as the USGS' $\mathrm{K}_{\mathrm{a}}$-band radar sensors over tidal rivers and creeks throughout the City of Virginia Beach [4]. A later paper will evaluate the differences between these sensor accuracies and types, fault tolerance in data transmissions, and solar power management schemes [6]. An initial comparison with a temporary rapid deployment gauge established by the USGS allowed for a



Figure 4. StormSense's information flow to guide Hampton Roads' data ingestion efforts to advise predictive flood models. 
favorable short-term data comparison with Norfolk's LoRaWAN sensor collocated there during a nine-day overlap period during Hurricane Maria [4].

\section{B. Water Level Sensor Accuracies and Costs}

After an evaluation period of 6-9 months, these collocated StormSense sensors will be relocated to unique monitoring locations in Virginia Beach. A small number of white papers and vendor brochures evaluate the accuracies of the ultrasonic and $\mathrm{K}_{\mathrm{a}}$-band radar sensors in laboratories or for the application of level monitoring of water treatment reservoirs or chemical vats. However, these are not comparable to tidal water bodies or areas with significant wave action, such as during the extratropical storm surge events presented in this study during Hurricanes Jose and Maria [6]. A cursory comparison from the initial deployments of the sensors in Summer 2017 revealed that the ultrasonic sonar units are from Valarm are accurate in the lab to a Root Mean Squared Error (RMSE) of $\pm 5 \mathrm{~mm}$, and accurate in the field to an average of $\pm 18 \mathrm{~mm}$, while the two $\mathrm{K}_{\mathrm{a}}$-band radar sensors in Newport News are accurate in the lab to $\pm 1 \mathrm{~mm}$ and accurate as deployed in the field to $\pm 9 \mathrm{~mm}$ [7]. The cost to purchase a solar-powered cellular transmission station was approximately $\$ 3000 /$ each for the ultrasonic sensors, and $\$ 4400 /$ each to purchase the $\mathrm{K}_{\mathrm{a}}$-band radar units [4]. The street inundation sensors employed in Norfolk through the vendor, Green Stream, are accurate in the lab to approximately $\pm 15 \mathrm{~mm}$, and accurate in the field $\pm 45 \mathrm{~mm}$, and sensors were purchased for $\$ 400 /$ each, plus the cost of the LoRa transmission gateway, which has an effective transmission range of approximately one mile, less the distances occluded by high-rise buildings [7].

\section{Water Level Sensor Data Comparisons}

A comparison of the five new street inundation sensors and one water level sensor in Norfolk, and eight new water level sensors in Newport News were used to temporally and vertically validate a street-level hydrodynamic model's predictions during the offshore passage of Hurricanes Jose and Maria, which detected increased water levels in Hampton Roads by $76.2 \mathrm{~cm}$ (2.5 ft.) and $60.9 \mathrm{~cm}$ (2 ft.), respectively. These six gauges resulted in an aggregate vertical RMSE of $\pm 8.93 \mathrm{~cm}$ over a 72-hour Hurricane Jose model forecast simulation [4].

The seven gauges present during Hurricane Maria (including the USGS rapid deployment gauge installed from 9/219/29/2017) yielded a more favorable aggregate RMSE of $\pm 6.28 \mathrm{~cm}$ when compared with the model. Both storms produced minimal surge-related coastal flooding, yet inundation impacts were equally profound in some tidalconnected inland areas, making the comparison with Norfolk's new street inundation sensors interesting to observe and practical for verification of inland inundation extents and depths. USGS measurements temporarily co-located at the same site during Hurricane Maria's passage were used to apply a vertical adjustment of $+4.5 \mathrm{~cm}(0.15 \mathrm{ft}$.), based upon the Mean Absolute Error (MAE) as an offset, to improve the Root
Mean Squared Error (RMSE) metric for this event, and likely for many events in the future [4]. This change resulted in an improvement in sensor estimated RMSE from 6.08 to $0.71 \mathrm{~cm}$, a difference of $5.37 \mathrm{~cm}(0.17 \mathrm{ft}$. $)$.

\section{DISCUSSION AND CONCLUSIONS}

In the future, smart city systems could evaluate tenable candidate blueprint solutions for flood-related problems, whether they be attributed to storm surge, heavy rainfall, or tides, as was the case during the offshore passage of 2017 Hurricanes Jose and Maria, using a decision matrix. This could help key decision-makers in areas analogous to Hampton Roads, VA, make informed decisions regarding how floodrelated solutions could be best addressed in their region. The new StormSense water level sensor network is being integrated into Tidewatch to create a regional resilience monitoring network to directly address a key recommendation from the area's Intergovernmental Pilot Project [3,25].

StormSense's value can be inherently measured in: time, money, and potential lives saved. For evidence of this, the project and the region's media partners asked citizens to put the new water level sensors and flood model predictions to the test during the 2017 king tide floods on 5 November, 2017. 500+ volunteers from 12 cities and counties helped to map 53,000+ GPS-recorded high water marks and collected 1,200+ geotagged photographs of flooding in Hampton Roads using a mobile app called Sea Level Rise [26]. Overall, the event revealed that the new StormSense sensors and model were vertically accurate within a root mean squared error of $2.21 \mathrm{~cm}$ and horizontally accurate within a mean spatial distance difference of $13.1 \mathrm{ft}$. [6,26].



Figure 5. Examples from three StormSense ultrasonic sonar sensors co-located in the field adjacent to USGS $\mathrm{K}_{\mathrm{a}}$-Band radar sensors in Virginia Beach for direct comparison of monitoring accuracy. These sensors will temporarily be stationed adjacent to each other for a period of 6-9 months to provide a long term data record for comparison of water level measurements, data transmission speeds, and solar power efficiency. Figure adapted from [4]. 
As sea levels rise, it is likely that this will continue to become an ever more pervasive issue. Analysis of the local sea level trend from the longest period record in Hampton Roads at Sewells Point in the City of Norfolk depicts a long-term linear increase in mean sea level of $4.59 \pm 0.23 \mathrm{~mm} /$ year since its establishment in 1928 [27]. The data from a new sea level trend study conducted at VIMS focuses on trends since the Anthropocene (1969-present)[28] to suggest that rising sea levels will inevitably exacerbate flooding conditions from storm events in the nearer-future than initially projected by the IPCC's fifth assessment report, leading to a linear increase in mean sea-level of $0.29 \mathrm{~m}$ by $2050[27,28]$. When considering a quadratic fit of these data, the curve suggests an elevated trend of $0.49 \mathrm{~m}$ by 2050 [28]. Cities, counties, town governments, local institutions, and private contractors, provide myriad solutions, each of which must be evaluated in its own way, and the subsequent presentation of their flood data ultimately impact their efficacy as a warning. Also, provision of these serviceable flooding solutions often impacts the availability of other services citizens rely upon. Thus, this establishment of StormSense's flood monitoring blueprint is designed to aid other communities in mitigating adverse inundation impacts.

\section{ACKNOWLEDGMENTS}

The authors would like to thank Mike Ashe, Wade Gerze, Frank James, and the Newport News Public Works department and Oceaneering, Inc. for their efforts in installing, calibrating, and maintaining the water level sensors. The sensors were purchased from Valarm through funding assistance graciously provided by NIST and VDEM. We thank the reviewers whose conscientious comments improved this paper in many ways during the peer-review process. Portions of this publication and research effort are made possible through the help and support of NIST via federal award \#70NANB16H277. This paper is Contribution No. 3734 of the Virginia Institute of Marine Science, College of William \& Mary. Official contribution of the National Institute of Standards and Technology; not subject to copyright in the United States. Certain commercial products are identified in order to adequately specify the procedure; this does not imply endorsement or recommendation by NIST, nor does it imply that such products are necessarily the best available for the purpose.

\section{LITERATURE CITED}

[1] Rhee, S. 2016. Catalyzing the Internet of Things and Smart Cities: Global City Teams Challenge. SCOPE '16 Science of Smart City Operations and Platforms Engineering in partnership with Global City Teams Challenge (GCTC), p.1.

[2] Casulli, V. 2015. A conservative semi-implicit method for coupled surfacesubsurface flows in regional scale, International Journal for Numerical Methods in Fluids, 79(4):199-214.

[3] Loftis, J.D., Molly Mitchell, Larry Atkinson, Ben Hamlington, Thomas R. Allen, David Forrest, Teresa Updyke, Navid Tahvildari, David Bekaert, and Mark Bushnell. 2018. Integrated Ocean, Earth and Atmospheric Observations in Hampton Roads, Virginia. Marine Technology Society Journal, 52(2), (In Press).

[4] Loftis, J.D., Forrest, D., Katragadda, S., Spencer, K., Organski, T., Nguyen, C., and Rhee, S. 2018. StormSense: A New Integrated Network of IoT Water Level Sensors in the Smart Cities of Hampton Roads, VA. Marine Technology Society Journal, 52(2), (In Press).
[5] Loftis, J.D., Wang, H.V. \& Forrest, D.R. 2016. Street-Level Inundation Modeling of Hurricanes Matthew and Hermine and Emerging Flood Monitoring Methods in Hampton Roads. William \& Mary Publish. URL

[6] Loftis, J.D., Wang, H.V. \& Forrest, D.R. 2017. Catch the King Tide with StormSense on Nov. 5th: How You Can Help Crowd-Source Tidal Flood Event Calibrations for Hampton Roads' Newest Water Level Sensors. William \& Mary Publish. URL

[7] Loftis, J.D., Wang, H., Forrest, D., Rhee, S., Nguyen, C. 2017. Emerging Flood Model Validation Frameworks for Street-Level Inundation Modeling with StormSense. SCOPE '17 Science of Smart City Operations and Platforms Engineering, 2(1), 13-18.

[8] Replicable Smart City Technologies (RSCT). 2016. Federal Funding Opportuninty Announcement: RSCT Cooperative Agreement Program. URL

[9] Sweet, W.V., Park, J., Marra, J.J., Zervas, C. \& Gill, S. 2014. Sea level rise and nuisance flood frequency changes around the United States, in NOAA Technical Report NOS COOPS 73, 53 pp.

[10] Ezer, T. \& Atkinson, L.P. 2014. Accelerated flooding along the US East Coast: on the impact of sea-level rise, tides, storms, the Gulf Stream, and the North Atlantic oscillations. Earth's Future. 2(8):362-382. URL

[11] Ezer, T.\& Atkinson, L.P. 2017. On the predictability of high water level along the U.S. East Coast: can the Florida Current measurement be an indicator for flooding caused by remote forcing?, Ocean Dynamics, 67(6): 751-766.

[12] VanHoutven, G., Depro, B., Lapidus, D., Allpress, J. \& Lord, B. 2016. Costs of Doing Nothing: Economic Consequences of Not Adapting to Sea Level Rise in the Hampton Roads Region. Virginia Coastal Policy Center, College of William \& Mary Law School Report. URL

[13] Lawlor, J. 2012. City Line Apartments: flood prone and no solutions in sight. Daily Press, August 29, 2012. URL

[14] Alley, R.B. 2017. Letter regarding flooding on Newmarket Creek and City Line Apartments from Newport News Fire Chief. Personal Correspondence. URL

[15] Smith, H. 2016. After yet another City Line Apartments flood, FEMA steps in to help. Daily Press, October 12, 2016. URL

[16] FEMA. 2016. National Flood Insurance Program coverage isn't the same as homeowner insurance or FEMA assistance, Virginia Beach, Dec. 5, 2016. URL

[17] Bannan, B., Burbridge, J., Dunaway, M., Skidmore, D., Brooks, D., Crane, T., DiBiase, R., Dubrow, S., Hopingardner, P., Icenhour, T., Namuduri, K., Osborn, C., Phool, T.S., Purohit, H. \& Thomas, G. 2017. Blueprint for Smart Public Safety in Connected Communities: Initiative of the Global City Teams Challenge. URL

[18] Wahl, T., Jain, S., Bender, J., Meyers, S.D. \& Luther, M.E. 2015. Increasing risk of compound flooding from storm surge and rainfall for major US cities, Nat. Clim. Change, doi:10.1038/nclimate2736.

[19] Loftis, J.D., Wang, H.V., DeYoung, R.J. \& Ball, W.B. 2016. Using Lidar Elevation Data to Develop a Topobathymetric Digital Elevation Model for SubGrid Inundation Modeling at Langley Research Center, Journal of Coastal Research, Special Issue 76:134-148.

[20] Loftis, J.D., Wang, H.V. \& DeYoung, R.J. 2013. Storm Surge and Inundation Modeling in the Back River Watershed for NASA Langley Research Center, NASA Technical Report, NASA/TM-2013-218046.

[21] Loftis, J.D. 2014. Development of a Large-Scale Storm Surge and High-Resolution Sub-Grid Inundation Model for Coastal Flooding Applications: A Case Study during Hurricane Sandy, Ph.D. Dissertation, College of William \& Mary. pp. 218.

[22] Wang, H., Loftis, J.D., Liu, Z., Forrest, D. \& Zhang, J. 2014. Storm Surge and SubGrid Inundation Modeling in New York City during Hurricane Sandy. Journal of Marine Sci. and Eng., 2(1), 226-246.

[23] Wang, H., Loftis, J.D., Forrest, D., Smith, W. \& Stamey, B. 2015. Modeling Storm Surge and inundation in Washington, D.C., during Hurricane Isabel and the 1936 Potomac River Great Flood. Journal of Marine Sci. and Eng., 3(3), 607-629.

[24] Loftis, J.D., Wang, H.V., Hamilton, S.E. \& Forrest, D.R. 2018. Combination of Lidar Elevations, Bathymetric Data, and Urban Infrastructure in a Sub-Grid Model for Predicting Inundation in New York City during Hurricane Sandy. Computers, Environment, and Urban Systems (In Review).

[25] Steinhilber, E.E., Boswell, M., Considine, C. \& Mast, L. 2016. Hampton Roads Sea Level Rise Preparedness and Resilience Intergovernmental Pilot Project. Phase 2 Report: Recommendations, Accomplishments and Lessons. URL

[26] Loftis, J.D. 2017. "Catch the King" Tide Thank You and Review. CCRFR Thank You and Review Community Event at ODU, Dec. 13, 2017. Pres. 41. URL

[27] Mitchell, M., Hershner, C., Herman, J., Schatt, J., Mason, P., Eggington, E. \& Stiles, W.S. 2013. Recurrent Flooding Study for Tidewater Virginia, Report submitted to the Virginia General Assembly, 135-150: URL

[28] Boon, J.D., Mitchell, M., Loftis, J.D. \& Malmquist, D.M. 2018. Anthropocene Sea Level Change: A History of Recent Trends Observed in the U.S. East, Gulf and West Coast Regions. VIMS Special Report in Applied Marine Science and Ocean Engineering (SRAMSOE). No. 467. VIMS, College of William \& Mary. URL 